\title{
GUBERNAMENTALIDAD, PEDAGOGÍA NEUTRA Y (DES)PROFESIONALIZACIÓN DOCENTE
}

\author{
Andrea Ruffinelli ${ }^{1}$
}

RESUMEN: Ensayo que analiza la tensión que experimenta en Chile el carácter profesional de la formación y del ejercicio docente en un sistema económico, sociopolítico y educativo neoliberal, sistema que aplica los principios del mercado y el gerencialismo para controlar las mentes de los individuos - 'gubernamentalidad' - mediante tecnologías performativas de la conducta. Éstas son capaces de hacer reconocer decisiones inducidas como personales, reduciendo el campo de acción docente a las prescripciones aparentemente desprovistas de contenido ideológico o político, que estimulan una pedagogía neutra e higiénica, orientada al objetivo impuesto externamente. Un sistema cuyo paradojal correlato discursivo es la profesionalización del rol docente.

Palabras clave: Gubernamentalidad. Performatividad. Profesionalización docente. Pedagogía neutra.

\section{GOVERNMENTALITY, NEUTRAL PEDAGOGY, AND TEACHER'S (DE)PROFESSIONALIZATION}

\begin{abstract}
This essay analyzes the stress experienced by the professional nature of teacher's training and practice in an economic, sociopolitical and educational system in Chile. This applies the principles of the market and managerialism to control the minds of individuals 'governmentality' - by using behavior performative technologies. These methods are able to recognize personal-induced decisions, thus decreasing the teaching scope to requirements that apparently do not have ideological or political content, stimulating a neutral and hygienic pedagogy, which is oriented to the externally imposed goal. A system whose paradoxical discursive correlate is the professionalization of the teaching role.
\end{abstract}

Keywords: Governmentality. Performativity. Teacher's professionalization. Neutral pedagogy.

\footnotetext{
${ }^{1}$ Universidad Alberto Hurtado - Santiago, Chile. E-mail: aruffine@uahurtado.cl DOI: 10.1590/ES0101-73302016139074
} 


\title{
GOUVERNEMENTALITÉ, PÉDAGOGIE NEUTRE ET (DÉ)PROFESSIONNALISATION DES ENSEIGNANTS
}

\begin{abstract}
RÉSUMÉ: Essai que analyse la tension au Chili associée à l'aspect professionnel de la théorie d'enseigner et le travail pratique des enseignants dans un système économique, sociopolitique et éducatif néolibéral qui utilise les principes du marché et du management pour contrôler les mentalités des individus - gouvernementalité — parmi des technologies performatives de la conduites. Ces méthodes sont capables de faire reconnaitre décisions personnelles qui ont été induites, réduisant ainsi le champs d'action des enseignants a prescriptions apparemment dépourvus de contenu idéologique ou politique qui stimulent une pédagogie neutre e hygiénique orientée vers un but imposé de façon externe. Un système dans lequel le paradoxal corrélat discursif est la professionnalisation du rôle de l'enseignant.
\end{abstract}

Mots-clés : Gouvernementalité. Performativité. Professionnalisation de l'enseignant. Pédagogie neutre.

\section{Introducción}

$\mathrm{E}$

1 texto aborda el concepto de gubernamentalidad planteado por Foucault (2007), estableciendo vinculaciones entre dicha forma de gobernar que opta por el control de las mentes de los sujetos - y el escenario educativo chileno, basándose en el tipo de reforma educativa implementada y profundizando en algunos efectos de la gubernamentalidad y del gerencialismo asociado, en tanto herramienta 'higienizadora' de la pedagogía desde el control de la gestión sobre la autonomía, planteando la paradoja de un sistema que tiene un discurso profesional para referirse a sus docentes, pero que en la práctica los desprofesionaliza. Esta paradoja se levanta por medio del análisis de las distintas políticas docentes implementadas desde la dictadura cívico militar y hasta antes de la promulgación de la nueva ley de desarrollo profesional docente en 2016.

\section{El concepto de gubernamentalidad y la tensión con la profesionalización docente}

Foucault (2007) elaboró una teoría del Estado en que se desplaza la idea de gobernar al pueblo por la de gobernar las conductas de los individuos: la gubernamentalidad, una perspectiva que propone estudiar al Estado trascendiendo a las instituciones de gobierno, y concibiéndolo como un producto determinado por otros procesos históricos, sociales y políticos (FOUCAULT, 2007). Esta teoría 
desarrolla un marco analítico que abstrae las relaciones de poder de la institución y las observa desde la perspectiva de las tecnologías.

El gobierno de la conducta de los sujetos se lleva a cabo mediante la diseminación de los dispositivos de seguridad a través del tejido social, entendidos como tecnologías de gobierno que toman la forma de instrumentos o instituciones para el ejercicio del poder, cuyo objetivo es normalizar y controlar las conductas de los individuos para dominar una realidad sin intervenirla directamente, sino subrepticiamente.

Entre las técnicas de dominación utilizadas se encuentran las del 'yo', que interactúan con los dispositivos de seguridad mencionados y se refieren a la actuación del individuo sobre sí mismo y a la circularidad que se genera cuando son integradas en las estructuras de coerción y dominación, encarnadas en los dispositivos de seguridad (FOUCAULT, 2007).

Bajo esta mirada, la no intervención directa del Estado es una técnica gubernamental que construye un tinglado capaz de transferir las operaciones del gobierno a los actores no estatales, en una opción deliberada por una forma de administración sustentada en responsabilidades individuales, privatización del riesgo y mercado, que trasciende a los más diversos ámbitos sociales y, con ello, al educativo (LEMKE, 2007).

Esa nueva comprensión del Estado, propia de las economías neoliberales de Occidente (LEMKE, 2007), ubica el foco en la gestión, concepto que en los espacios educativos ha llegado a ocupar un nivel de culto compartido masivamente en la asunción que se trata de la indiscutida mejor forma de conducir las organizaciones educacionales (BALL, 1993).

En este modelo se implementa un estilo burocrático que controla el trabajo de los docentes por medio de las herramientas propias de la lógica productiva industrial y de la competencia, en cuyos procesos de toma de decisiones los profesores no tienen injerencia (BALL, 1993). Ellos son 'objetos' y no 'sujetos' de la política, situación que tensiona los elementos constitutivos de la construcción de la autonomía profesional: el perfil, las decisiones y las actitudes profesionales (LANG, 2006).

Se trata de un enfoque que impone un particular modo de entender la profesionalización, sustentado en la idea que el mejoramiento educativo sólo es posible si los docentes se alinean a las reglas del mercado y las encarnan, ocupándose en buscar su perfeccionamiento continuo y el incremento de sus remuneraciones, mientras los expertos (ministerios) se responsabilizan por definir los criterios de selección de los estudiantes de pedagogía, de los programas de formación inicial y continua, del acceso e itinerario de la carrera docente, de la evaluación del desempeño docente y de los incentivos al desempeño (TENTI, 2004). 


\section{Las tensiones del escenario educativo mundial, sus efectos en la profesionalidad docente y las elecciones del sistema chileno}

La masificación del sistema escolar en la sociedad del conocimiento ha puesto a las naciones en la encrucijada de optar por reformas educativas fundamentalmente de dos tipos: basadas en la equidad y el desarrollo de capacidades, y sustentadas en la rendición de cuentas e incentivos (DARLING-HAMMOND, 2012).

Las primeras reformas configuran sistemas educativos intelectualmente desafiantes, basados en un currículo reflexivo y en la promoción de habilidades complejas y colaborativas, que son avaladas por un alto nivel de calificación de sus docentes y de sistemas de rendición de cuentas orientados a la evaluación permanente, con fines de retroalimentación y mejora de las capacidades.

El otro grupo de reformas se vincula a la gobernanza escolar que discursivamente se refiere a los docentes como personas libres y capaces de construir su carrera, promoviendo la autonomía y autorregulación profesional, pero condicionándolos mediante estrategias de mercado (FELDFEBER, 2007). Es así como incorporan exámenes nacionales, individualizan la docencia pagando por mérito, reducen la inversión en formación de profesores y la desvinculan de la academia, atraen personas sin preparación docente a la enseñanza y las estimulan a competir con sus pares. Se somete a estudiantes y profesores a mediciones con el fin de premiar/castigar docentes y escuelas, generando un trabajo escolar orientado a responder tales pruebas, con el consiguiente estrechamiento del currículum y desvalorización de los docentes.

Chile es uno de los países que ha orientado su reforma educativa en este último sentido (DARLING-HAMMOND, 2012), y si bien es cierto que el campo de acción docente tiene una especificidad propia, dentro de la que debe actuar el profesor, sustentado en su preparación y juicio experto (ABBOTT, 1988), es también vulnerable a los cambios en las estructuras fundamentales como el currículum, los modos de administrar el sistema educativo, y al nivel de prescripción externa sobre cómo ejercer la práctica docente (TENTI, 2006; DAY; GU, 2010).

En este escenario, surge como correlato la tensión por la definición de los sentidos y contenidos de la profesionalización. Algunos autores plantean modelos identitarios similares. El modelo del 'management' (DUBET et al., 1999), o 'tecnológico' en palabras de Lang (2006), ligado a los principios de la burocracia: eficiencia, estandarización de objetivos, procedimientos y mediciones con resultados esperados predefinidos, entendiendo al profesor como un tecnócrata, con un rol de didacta, siendo clave el componente científico técnico del oficio.

Como contrapunto, describen el 'modelo republicano' (DUBET et al., 1999), u 'orgánico' para Lang (2006), vinculado a lógicas basadas en consideraciones culturales, ético/morales y políticas, sustentadas en la confianza en la auto-regulación 
docente derivada de la autonomía profesional y de la responsabilidad del colectivo docente. Entiende al profesor como un profesional 'clínico', capaz de diagnosticar, definir estrategias de diversa índole y fines (no sólo instrumentales) y generar resultados de diversa naturaleza. Su rol es social y político, promotor de las causas de los derechos humanos universales.

Desde las perspectivas de estas racionalidades, veremos cómo en Chile se ha optado por el primer modelo.

\section{La paradoja del sistema que plantea desafíos profesionales a sus docentes y simultáneamente los desprofesionaliza}

En Chile, el discurso oficial - extraído desde los dos instrumentos que norman la práctica docente: los "Estándares Orientadores para Egresados de Carreras Pedagógicas" (MINISTERIO DE EDUCACIÓN, 2011; 2012) y el "Marco para la Buena Enseñanza” (MINISTERIO DE EDUCACIÓN, 2008) — releva una visión del profesor que se ajusta al primer grupo de reformas, concibiéndolo en una categoría profesional. Sin embargo, este acto cohabita - y lo viene haciendo desde hace unos 40 años - con políticas e instrumentos que apuntan en sentido contrario, y que, paradójicamente, son justificadas como medios para alcanzar la profesionalización de los docentes y el mejoramiento del aprendizaje de los estudiantes.

En este punto, es preciso señalar que la comprensión de la docencia como profesión tanto en Chile como en otros países de Latinoamérica recién se fragua, y lo que existe es una tradición identitaria magisterial funcionaria y técnica, que ha transitado desde el 'apostolado' en los inicios de la escuela a manos de las iglesias, para luego identificarse con la comprensión de funcionario público con la irrupción del rol del Estado en la educación. Más tarde, en la fase de la masificación educativa, se ha venido asumiendo fuertemente el rol técnico antes descrito, que sólo en las últimas décadas ha iniciado un camino hacia una comprensión 'profesional' (NÚNEZZ, 2004).

Los actuales estándares nacionales para egresados de las carreras de educación básica y media señalan que los docentes son profesionales que deben contar con habilidades y actitudes para el trabajo colaborativo, autonomía, flexibilidad, capacidad de innovar, disposición al cambio y proactividad, además de una sólida formación en valores y comportamiento ético, manejo de las comunicaciones oral y escrita en la lengua materna y segunda lengua, habilidades en uso de tecnologías de información y comunicación (TIC), un nivel cultural propio de mundo globalizado, así como espíritu de superación para ocuparse de su desarrollo personal y profesional permanente (MINISTERIO DE EDUCACIÓN, 2012). En tanto, el "Marco para la Buena Enseñanza" señala que el profesor es un profesional capaz de: preparar apropiadamente la enseńanza, crear un ambiente propicio para el aprendizaje, enseñar a sus estudiantes de modo que aprendan, y asumir sus 
responsabilidades profesionales, reflexionando consciente y sistemáticamente sobre su práctica y reformulándola a partir de dicha reflexión (MINISTERIO DE EDUCACIÓN, 2008).

Este prototipo de desempeño profesional es tensionado por un contexto socioeconómico, político y cultural que demanda a sus profesores no sólo rápidas y certeras adecuaciones ante los cambios vertiginosos de la sociedad, de las políticas y del currículum que deben enseñar, sino también proactividad en el ejercicio en condiciones difíciles, con remuneraciones inferiores a profesiones comparables, escaso tiempo para preparar y evaluar la enseñanza, enfrentamiento de las exigencias de manejo cabal de la diversidad, vulnerabilidad y desmotivación de sus estudiantes y familias, de las progresivas exigencias evaluativas ministeriales y de las demandas sociales que exigen estar siempre actualizado, demandas que se incrementan de manera inversamente proporcional a las condiciones ofrecidas para alcanzar las capacidades necesarias a la valoración social de la profesión, haciendo más difícil lo que ya lo es (ÁVALOS, 2013).

Desde esta perspectiva, el rol docente trasciende con mucho la enseñanza exitosa de un currículum explícito, debiendo hacerse cargo de los problemas estructurales de la sociedad y, pese a los cuales, se le demanda lograr aprendizajes integrales de calidad con equidad, cargando con la responsabilidad de desempeñar la profesión que ha sido identificada como la clave para el desarrollo del país, en una situación que difiere sustantivamente del contexto educativo antes de la escolarización plena.

Sin embargo, las expectativas y exigencias de la más alta sofisticación profesional surgen en un escenario que venía avanzando por décadas en la implementación de políticas gerencialistas conscientemente articuladas, que implican nociones de profesionalización ligadas a incentivos individuales por resultados, tensionando la identidad docente histórica más vinculada a la ética del servicio público (BELLEI, 2001). Esa situación ha derivado en un sentimiento de desprofesionalización y desvalorización docente, tanto en dictadura como en democracia, que han incluido:

- En dictadura: Cierre de las escuelas normales que hasta 1974 formaron a los profesores; redefinición del rol del Estado en la educación, asumiendo que el fin de la educación básica sería capacitar para ser buenos trabajadores, ciudadanos y patriotas, y autorizando a los establecimientos a focalizarse en la alfabetización y en el cálculo básico, lo que redunda en el constreñimiento de la formación docente y del currículum escolar, incluyendo la eliminación de contenidos que promovieran el pensamiento crítico en todos los niveles del sistema educativo. En 1981, se elimina el carácter universitario a la formación docente (ARÉVALO et al., 2013) - que se recupera recién el año 2015 - y, simultáneamente, se entrega la formación de profesores a los principios de un mercado fuerte- 
mente des-regulado, que deriva en una precarización de la calidad de la formación impartida (COX et al., 2010).

En 1988 se levantó un pilar fundamental de la gubernamentalidad educativa actual, la Prueba SIMCE ${ }^{1}$. Esta es una prueba nacional censal, anual y estandarizada de lápiz y papel, para escolares de diferentes niveles educativos, presentándose estos resultados como indicadores de calidad de las escuelas e identificando a los establecimientos educativos en la comunicación de sus resultados, bajo la forma de rankings, hasta la medición del año 2012, que responsabiliza a las escuelas por los resultados, pese a que se asocian consistentemente al origen socioeconómico de los estudiantes y no a los efectos de su escolarización. Esta información se hace pública, con el objeto de estimular a la competencia entre escuelas para lograr más matrícula, y, según la experiencia de los países que han aplicado la estrategia, derivan en estrechamiento curricular y en una formación y ejercicio docente acotado a prescripciones que garanticen buen rendimiento en estas pruebas, desvalorización del rol docente y deterioro del clima escolar (ALEXANDER, 2010; 2011; HARGREAVES; FULLAN, 2012).

- En democracia: La esencia gerencialista del modelo societal y educativo no sólo no fue modificada en democracia, sino que fue sofisticada (OSBORNE; GAEBLER, 1993). Muestras de eso son el crecimiento explosivo y des-regulado de las carreras de pedagogía, alcanzando a más de 1.500 programas diferentes y más de 120.000 estudiantes, con un incremento de la matrícula superior al $200 \%$ en diez años (COX et al., 2010). Es un mercado que se desarrolla en muy heterogéneas condiciones de exigencias de ingreso, egreso y oportunidades formativas, sobre un escenario básico de precariedad, entre cuyos estudiantes uno de cada tres ni siquiera rindió la prueba de selección universitaria, y entre los que lo hicieron, más del $70 \%$ logró menos de 500 puntos - el promedio del instrumento - además de ofrecerse unas perspectivas laborales difícilmente atractivas, al ser una de las profesiones peor remuneradas (MINISTERIO DE EDUCACIÓN, 2013).

A nivel escolar, la reforma curricular de 1996 fija el centro en lenguaje y matemática, reforzando el carácter alfabetizador de la educación básica y el inicio del doble estándar en el año 2009 con la "Ley General de la Educación", que determina nuevos objetivos orientados a la formación integral como sujetos autónomos y reflexivos, elaborando unas Bases Curriculares que responden a este sentido integral, trascendiendo al foco en disciplinas básicas (2012). Sin embargo, el sistema de evaluación vuelve a relevar como prioridad lenguaje y matemática (ARÉVALO et al., 2013). 
En 2003, se implementó la política docente ícono del gerencialismo: la evaluación y los incentivos al desempeño docente, orientando explícitamente a los profesores hacia el modelo de profesionalismo propio de este enfoque, que es percibido por ellos como ajeno a su rol (SISTO, 2011). Políticas de profesionalización similares, sustentadas en el desempeño, se encuentran con frecuencia también en las reformas educativas de otros países latinoamericanos (TENTI, 2006) y en Europa (GUNTER, 2008).

La principal tensión docente frente a esta evaluación estriba en la reconceptualización que hace de su trabajo, privilegiando el desempeño individual por sobre el colectivo, estandarizando y prescribiendo, y con ello restringiendo la posibilidad de vincularse a su práctica habitual, debiendo mostrar evidencias ajustadas a categorías prefijadas (SISTO, 2011).

En la misma línea del doble discurso, los estándares, la rendición de cuentas y el control, surgió en 2008 otro pilar de la gubernamentalidad: la evaluación del egreso a la profesión docente, la prueba anual INICIA ${ }^{2}$, de lápiz y papel, que mide a través de preguntas de selección múltiple, fundamentalmente, conocimientos disciplinarios y pedagógicos de los estudiantes de pedagogía al terminar su proceso formativo, restringiendo en este acto el rol docente a estos dominios y estas formas de evidencia de sus saberes, que en cuatro de sus versiones (años 2010 a 2013) identifica a las instituciones y las rankea. La prueba tiene el objetivo de controlar indirectamente la conducta de las instituciones formadoras, en el sentido de persuadirlas a focalizar sus esfuerzos en obtener buenos resultados para captar más y mejores estudiantes, misma situación en la que ya tienen vasta experiencia las escuelas a causa de la prueba SIMCE, haciendo enfrentar a las instituciones e individuos las consecuencias de unos resultados que se asumen de su exclusiva responsabilidad, reservándose para el Estado el rol de informador.

Como corolario para la desprofesionalización, en 2009, se promulgó un artículo de ley (MINISTERIO DE EDUCACIÓN, 2009) que permite a titulados de cualquier carrera de ocho semestres de duración hacer clases en la enseñanza media o secundaria, reconociendo que no se requieren saberes profesionales específicos para el ejercicio de la docencia (COX et al., 2010). Es un nuevo instrumento de control que disemina la idea de un profesor sin estatus profesional, lo cual difiere del discurso oficial que le advierte que será juzgado con los más altos estándares profesionales por haberle entregado la responsabilidad de formar a sus ciudadanos, pero en la práctica, implementa políticas que desvalorizan y miden a los profesores a partir de un concepto restringido de la docencia.

En suma, cohabitan un discurso oficial promotor de profesionales reflexivos y críticos y medios de control en sentido inverso, un doble discurso, la paradoja de un sistema que desprofesionaliza y mantiene expectativas y exigencias de desempeño profesional. 
Ha quedado fuera de este análisis un hito muy reciente en la historia de la política educativa en democracia: la promulgación de la nueva Ley de Desarrollo Profesional Docente (carrera docente) a inicios del año 2016, cuya discusión en torno a sus concepciones, vinculaciones con el modelo gerencialista y posibles efectos para la profesionalización merece un texto aparte.

\section{Los efectos de la gubernamentalidad en la profesionalidad docente}

El acceso democrático a carreras de pedagogía como argumento de reivindicación social - amparado por la des-regulación estatal — confunde el derecho a la educación con el derecho a ser profesor. Cuatro décadas de discurso aspiracional y meritocrático individual han convencido a un país que todos deben acceder a la educación superior para 'ser alguien'. Una de las alternativas más accesibles y de bajo costo (con financiamiento estatal) para eso, es la de estudiar pedagogía.

Cualquier universidad (y hasta el año 2014, cualquier Instituto Profesional) podía impartir una carrera pedagógica, contando o no con acreditación, y cualquiera podía estudiar pedagogía si pagaba por ello, disponiendo de una amplia gama de instituciones sin requisitos de ingreso, con programas formativos de dudosa calidad. Cuando se trata de instituciones acreditadas - que en Chile no es sinónimo de calidad —, la señal del Estado es que es indistinto estudiar en cualquiera de ellas, porque hay financiamiento para la diversidad de programas acreditados, y si hay alguna diferencia, el consumidor/cliente debe evaluarlo personalmente, haciendo uso de las asimétricas herramientas de discernimiento disponibles - como los resultados de la prueba INICIA, que, por sobre todo y al igual que SIMCE, da cuenta de las diferencias socioeconómicas de origen de los egresados y mide de modo altamente sesgado la calidad formativa de las instituciones, junto con hacer recaer toda la responsabilidad de la elección del programa sobre el postulante y su familia, sin responsabilidad alguna del Estado, como garante de un servicio educativo de calidad en la formación docente que financia.

Como parte del modelo, los instrumentos de la política arrojan sus resultados, que se espera sean usados para elegir escuela o programa formativo. La última evaluación nacional de desempeño docente en $2014^{3}$ — que se aplica sólo a profesores ejerciendo en el sector municipal — indicó que más del $20 \%$ de los evaluados no alcanza el nivel mínimo esperado, en tanto los resultados de las pruebas estandarizadas y restringidas de las que hemos venido hablando, como la prueba INICIA, son calificados por el propio programa homónimo como 'preocupantes'. Ellos muestran que la mayoría de los docentes recién egresados no cuenta con los saberes mínimos necesarios para desempeñarse idóneamente. Adicionalmente, los hallazgos del informe TEDS- $\mathrm{M}^{4}$ reportan que los docentes chilenos que se desempeñan en esta área son calificados en el ranking internacional en el lugar 15, de un total de 16 países participantes. Estos datos se 
suman a también precarios resultados nacionales e internacionales del aprendizaje escolar en pruebas estandarizadas como el SIMCE 5 , PISA ${ }^{6}$ y TIMSS ${ }^{7}$, que, tal como hemos señalado, ofrecen una visión parcial de lo que los estudiantes logran aprender en las escuelas.

Sin embargo, estas evaluaciones no consideran lo que aparece como más relevante en la interpretación de estos resultados - a la luz de las responsabilidades comprometidas en esta situación y de las oportunidades efectivas de aprender a enseñar que tuvieron estos profesores en las instituciones formadoras - a partir de itinerarios formativos que se hicieran cargo de la responsabilidad de aceptar postulantes, con capacidades insuficientes para el desafío.

Es decir, se observa un fustigamiento a los profesores como responsables individuales de los resultados educativos, omitiendo que fue este sistema nacional el que permitió aceptarlos como postulantes sin exigir requisitos; que permitió a las instituciones formadoras operar autónomamente, reguladas sólo por el mercado; que posibilitó a esas instituciones nutrirse del patrimonio económico público, y en muchos casos privado; que permitió el egreso y la titulación conforme a derecho de estos profesores, y que al final del periplo pone como piedra de tope un examen - que en el proceso de discusión de la recientemente aprobada Ley de Desarrollo Profesional Docente se promovía fuese vinculante a la posibilidad de ejercicio profesional, decisión que finalmente no se aprobó - , examen con la capacidad de determinar que todo el esfuerzo personal y económico fue en vano, y convencer de haber cometido un error personal, de que no se hizo el suficiente esfuerzo.

\section{Gerencialismo o la higienización de la pedagogía al servicio de la desprofesionalización}

En las páginas previas, se ha desarrollado la tensión entre una formación/ejercicio discursivamente orientados al desarrollo de profesionales capaces, críticos, reflexivos y unas demandas reales que reclaman técnicos funcionales a un sistema regido por el estándar. Uno que necesita docentes que implementen pasivamente planificaciones, clases y evaluaciones prescritas, definidas por los estándares de las pruebas externas; cruzadas por la urgencia de logros dadas las altas consecuencias, que a su vez se cruzan de manera subterránea con la demanda tácita de no formar profesores desde una perspectiva profesional, reflexiva y crítica, pues se constituirían en desestabilizadores no funcionales al engranaje del sistema basado en el gerencialismo.

En suma, un profesor desprovisto de capacidades profesionales - a las que se hace mención el "Marco para la Buena Enseñanza" (MINISTERIO DE EDUCACIÓN, 2008) y los "Estándares para Egresados de Pedagogía" (MINISTERIO DE EDUCACIÓN, 2012) —, amedrentado por las consecuencias vinculadas a unos logros impuestos externamente, convencido de una responsabi- 
lidad individual frente a tales logros y de que esos fines y medios son los apropiados para el desarrollo de sus estudiantes, es el ideal para este sistema, mientras, paradojalmente, los aprendizajes profesionales relativos a la preparación autónoma de la enseñanza, ajustada a las necesidades de desarrollo integral de unos estudiantes específicos, la implementación y evaluación de una enseñanza adecuada para ellos no tienen cabida, pues se ha determinado que hay otros objetivos que cumplir, en plazos siempre estrechos.

Entonces, aparece un elemento clave que otorga mayor retroalimentación al sistema: se presentan al gerencialismo y sus dispositivos de seguridad como ideológicamente neutros, inocuos, higienizantes por su carácter apolítico, exclusivamente orientados a la tarea, a la eficiencia, como si la eficiencia fuese un concepto técnico y neutro, asumiendo que es intrínsecamente positiva y deseable, y desconociendo los costos implicados en este proceso: sobrecarga laboral, estrés por supervisión y control constantes, pérdida de autonomía y confianza, disminución de participación en la toma de decisiones pedagógicas y deslegitimación del desarrollo profesional mediante el propio trabajo.

En este modelo, se asume la incompetencia del docente que debe ser dirigido por otros, los verdaderos expertos, perdiendo su influencia en la definición de la escuela. El currículum se constituye, de esta forma, en un sistema de distribución y los docentes son sus operarios o técnicos (BALL, 1993).

Como señaláramos, Foucault (1977) postuló que al gestionar de este modo se hace uso de la llamada 'tecnología moral', en el que el poder no radica en nadie en particular de modo completo, sino que todos están atrapados en este tinglado: quienes ejercen el poder y quienes se someten, es decir, una tecnología del 'yo', que induce a que las personas realicen - por sí mismos o con la ayuda de otros - operaciones sobre su conducta, a fin de lograr transformaciones que permitan alcanzar ciertos estados definidos por otros (FOUCAULT, 1988).

Thompson y Pascal (2012) identifican el 'gerencialismo' como una amenaza para la práctica reflexiva - propia de un profesional - en la medida en que el énfasis en el control de gestión (que implica falta de confianza y respeto por el supervisado) a costa de la autonomía profesional, genera un ambiente de trabajo adverso al aprendizaje reflexivo y, por extensión, a la profesionalización docente.

En palabras de Angus (1994), se trata de un ejemplo práctico de orientación funcionalista que escinde artificialmente la política del ejercicio docente, limitando su campo de acción, discernimiento y participación, defendiendo los beneficios de un ejercicio profesional de apariencia neutral e higiénica, que aleje al docente de las distracciones del contexto social, económico y político, del cuestionamiento a lo que le ha sido impuesto y que ha asumido como definición propia con la ayuda de los mecanismos de la gubernamentalidad, de manera que sólo quede espacio para concentrarse en lo que se ha definido como su función puntual: implementar la enseñanza del modo que se ha prescrito. 
Este esquema opera bajo la premisa que la mejor elección es no cuestionar lo encomendado, lo social ni mucho menos las condiciones políticas de su trabajo, pues una actitud neutra permite focalizar en la docencia y contribuir al mejoramiento educativo, perfeccionar su desempeño y el de sus estudiantes, en clave de arenga, omitiendo deliberadamente cuestionar las características de la sociedad, ni los resguardos que se hayan tomado para asegurar la calidad de la formación de profesores, ni las condiciones bajo las cuales se ejerza la docencia, naturalizando la restricción del campo y acción pedagógica al aula, que debe ser asumida desde prescripciones homogéneas y hegemónicas.

Se constituye así una invitación expresa a omitir poner los problemas educativos dentro de su contexto social más amplio - acción inherente a la histórica función social y política del oficio - en un doble juego, que por un lado responsabiliza a los educadores de casi todos los males sociales, y por otro, simultáneamente, niega los recursos para asumir con seriedad la educación, profundizando y haciendo estructurales en este acto las condiciones que dificultan el trabajo pedagógico. Esto se traduce en una espiral en que los docentes vuelven a ser culpables de todo, pese a que se constriñe su formación, campo de acción y condiciones de ejercicio profesional, y que en una suerte de acto psicomágico deben ser capaces de cambiar el mundo sin herramientas, desde una higienizada relación con el logro de altos estándares hasta una apología de la aceptación acrítica.

\section{A modo de conclusión: una síntesis del correlato de la gubernamentalidad y la acción educativa neutra e higiénica}

La opción por un sistema educativo construido bajo una racionalidad mercantil que se sustenta en estándares y rendición de cuentas vía mediciones e incentivos individuales externos, y bajo la convicción que las presiones, los premios y los castigos incrementarán y emparejarán los resultados de aprendizaje de los estudiantes, sin importar las consecuencias no deseadas, e incluso que los fines perseguidos (mejoramiento de aprendizajes) no sean alcanzados, muestran entre sus efectos la desprofesionalización de los docentes. Así es como se ha privilegiado la instalación de la competencia e incentivos pese a la evidencia sostenida que muestran los magros indicadores bajo su propio sistema de medición. En realidad, esos resultados no son el foco, sino la consolidación de una nueva forma de dominación, funcional al sistema social, económico y político imperante, sustentado en neoliberalismo, gubernamentalidad y gerencialismo educativo.

El poder ya no se encuentra centralizado, se disemina a través de las escuelas, sus autoridades y profesores. Se entrega a las instituciones escolares una autonomía cuyos márgenes son velada y rígidamente impuestos, dentro de los que se genera una falsa ilusión de libertad, en términos de un espacio generativo cuyo potenciamiento y desarrollo depende exclusivamente de su desempeño, que 
obviamente ha de estar alienado a la política central, que ya no es percibida como central, sino como una decisión autogestionada.

En este escenario, no se requieren estructuras sociales externas que regulen la conducta, pues ya están incorporadas en los agentes educativos que encarnan y diseminan esa estructura. Es lo que se ha denominado la 'tecnología del yo', en una suerte de autogobierno interiorizado, que naturaliza y organiza la práctica cotidiana desde una concepción gerencialista del sistema educativo.

Bajo esta perspectiva, un buen profesor es quien compite con sus colegas intra- e interescuela y obtiene mejores resultados en la prueba SIMCE que sus pares, mediante la aplicación pasiva y homogénea de prescripciones, las que no reconoce como imposición, sino ya está convencido de que es la forma apropiada de lograr ese objetivo, y de la legitimidad del modelo, ignorando contextos locales y nacionales, ocupado en aprender técnicas ideológicamente neutras que garanticen que su desempeño sea una ejecución lo más ajustada posible de lo que le ha sido encomendado dentro de ese espacio, un estrecho y ciego espacio de aula y sumisión.

En suma, es un sistema de doble estándar, que declara discursivamente la promoción de la formación de docentes profesionales y que, en la práctica, implementa políticas y una institucionalidad en sentido contrario, difundidas como instrumentos de apariencia ideológica neutral y generando un amplio cuerpo de representaciones que son aprendidas por los individuos. Estas representaciones - que resultan consistentes sólo en el contexto del discurso mercantil — dan lugar a una serie de argumentaciones que se introyectan y encarnan en los agentes, quienes asumen un discurso y prácticas impuestas, creyendo que se trata de una decisión personal conducente a fines deseables por todos. Estos agentes despliegan dispositivos de seguridad que no obligan a alinearse, sino que lo logran voluntariamente, formateando la conducta, y haciendo de este modo innecesario el control externo, pues cada quien se autocontrola usando los parámetros definidos por los que detentan el poder, y que han asumido como propios.

En este punto, es preciso recordar que la frecuentemente citada "desprofesionalización de los docentes" ha derivado - en otras latitudes — de las políticas públicas de control y estandarización, pero que en Chile la docencia históricamente no ha sido concebida con un carácter profesional, identidad que sólo reciente - y paradojalmente, como se ha argumentado en estas páginas — comienza a plantearse.

Con todo, como señala Tenti (2006), sería ingenuo - y contradictorio asumir que la definición del sentido y los contenidos de la profesionalización son susceptibles de una solución técnica, pues precisamente el problema está en el control de la autonomía docente. Desde esta perspectiva, parece necesario abordar posibles soluciones no sólo a partir de esta nueva comprensión profesional, sino atendiendo a que no tiene intrínsecamente un significado único, y el significado que se asuma debe encontrar un correlato coherente en las características de los procesos formativos y en las demandas reales del ejercicio docente, ambos sustentados en decisiones políticas que el trabajo investigativo futuro en este ámbito debe ayudar a develar y evaluar. 


\section{Notas}

1. Prueba que evalúa los resultados de aprendizaje de los establecimientos, evaluando el logro de los contenidos y habilidades del currículo vigente, en diferentes asignaturas o áreas de aprendizaje a través de una medición que se aplica a todos los estudiantes del país que cursan los niveles evaluados. Para más informaciones, visite: <http://www.agenciaeducacion.cl/simce/que-es-el-simce/>.

2. Visite el sitio para más informaciones: <http://www.evaluacioninicia.cl/>.

3. Visite el sitio para más informaciones: <http://portales.mineduc.cl/usuarios/mineducl doc/201506191506400.Resultados\%20finales\%202014.pdf>.

4. La evaluación TEDS-M, Teacher Education Study in Mathematics, de la International Association for the Evaluation of Educational Achievement (IEA), es un estudio comparativo internacional sobre la formación inicial docente en matemáticas para educación primaria y secundaria, desarrollado entre 2007 y 2008. Participaron 16 países: Estados Unidos, Alemania, Noruega, Polonia, la Federación Rusa, España, Suiza, Taiwán, Singapur, Tailandia, Malasia, Botsuana, Filipinas, Chile, Georgia y Omán, aunque este último no fue contabilizado en los resultados. En Chile, se aplicó sobre una muestra representativa de 1.400 estudiantes del último año de pedagogía en educación básica. Disponible en: <http://www.iea.nl/?id=20>.

5. Prueba aplicada por la Agencia Nacional de la Calidad de la Educación a estudiantes de distintos niveles de escolaridad en diferentes subsectores del currículum, de carácter censal en algunos y representativo en otros, destinada a informar sobre el desempeño de los estudiantes; evaluar el grado de cumplimiento de los Estándares de Aprendizaje y relacionarlos con el contexto escolar y social en que los alumnos aprenden, con el fin de contribuir a mejorar la calidad educativa. Disponible en: <http://www.agenciaeducacion.cl/simce/que-es-el-simce/>.

6. Program for International Student Assessment, que evalúa cada tres años las competencias de los estudiantes de 15 años en Lectura, Matemática y Ciencias, enfatizándose un área en cada ciclo.

7. Estudio Internacional de Tendencias en Matemática y Ciencias que desarrolla la IEA.

\section{Referencias}

ABBOTT, A. The system of professions: an essay on the division of expert labor. Chicago: The University of Chicago Press, 1988.

ALEXANDER, R. (ed.) Children, their world, their education. London: Routledge, 2010.

Evidence, rhetoric and collateral damage: the problematic pursuit of 'world class' standards'. Cambridge Journal of Education, v. 41, n. 3, p. 265-286, 2011.

ANGUS, L. Sociological analysis and educational management: the social context of the self managing school. British Journal of Sociology of Education, v. 15, n. 1, p. 79-91, 1994. 
ARÉVALO; A.; GYSLING, J.; REYES L. Bases para una propuesta de formación inicial docente de educación básica. Cuadernos de trabajo n. 1. Santiago, Chile: Departamento de Estudios Pedagógicos, Facultad de Filosofía y Humanidades, Universidad de Chile, 2013.

ÁVALOS, B. ¿Héroes o villanos? La profesión docente en Chile. Santiago, Chile: Editora Universitaria, 2013.

BALL, S. La gestión como tecnología moral. Un análisis ludista. En: BALL, S. (comp.) Foucault y la educación. Disciplinas y saber. Madrid: Morata, 1993. p. 155-168.

BELLEI, C. El talón de Aquiles de la reforma educativa. En: MARTINIC, S.; PARDO, M. (eds.) Economía politica de las reformas educativas en América Latina. Santiago, Chile: PREAL-CIDE, 2001. p. 129-146.

COX, C.; MECKES, L.; BASCOPÉ, M. La institucionalidad formadora de profesores en Chile en la década del 2000: velocidad del mercado y parsimonia de las políticas. Pensamiento Educativo, v. 46-47, p. 205-245, 2010.

DARLING-HAMMOND, L. Educar con calidad y equidad: los dilemas del siglo XXI. Santiago de Chile: Fundación Chile, 2012.

DAY, C.H.; GUY, Q. The new lives of teachers. Londres: Routledge, 2010.

DUBET, F.; BERGOUNIOUX, A.; DURU BELLAT, M. Le collège de l'an 2000: rapport à la ministre déléguée chargée de l'enseignement scolaire. Paris: Documentation Française, 1999.

FELDFEBER, M. La regulación de la formación y el trabajo docente: un análisis crítico de la "agenda educativa" en América Latina. Educação e Sociedade, v. 28, n. 99, p. 444465, 2007.

FOUCAULT, M. The eye of power. En: GORDON, C. (ed.) Power/ knowledge: selected interviews and other writings. Nueva York: Pantheon, 1977. p. 146-165.

Truth, power self: an interview. En: MARTIN, L.; GUTMAN, H.; HUTTON, R. (eds.) Technologies of the self. Londres: Tavistock, 1988. p. 141-150.

Nacimiento de la biopolitica. Curso en el Collège de France (1978-1979). Argentina: Fondo de Cultura Económica, 2007.

GUNTER, H., Policy and workforce reform in England. Educational Management Administration \& Leadership, v. 2, n. 36, p. 253-270, 2008.

HARGREAVES, A.; FULLAN, M. Professional capital. Transforming teaching in every school. Londres: Routledge, 2012.

LANG, V. La construcción social de las identidades profesionales de los docentes en Francia: enfoques históricos y sociológicos. En: TENTI, E. (comp.) El oficio de docente: vocación, trabajo y profesión en el siglo XXI. Buenos Aires: Siglo Veintiuno, 2006. p. 71-118.

LEMKE, T. Biopolitics: and advanced introduction. Nueva York y Londres: New York University Press, 2007. 
MINISTERIO DE EDUCACIÓN. Estándares orientadores para egresados de carreras de pedagogía en educación básica: estándares pedagógicos y disciplinarios. Santiago, Chile: Centro de Investigación Avanzada en Educación de la Universidad de Chile y el Centro de Estudios de Políticas y Prácticas en Educación de la Pontificia Universidad Católica de Chile, 2011. Disponible en: <http://www.cpeip.cl/usuarios/cpeip/File/2012/ librobasicaokdos.pdf>. Acceso en: 14 jun. 2016.

. Estándares orientadores para egresados de pedagogía en educación básica. Santiago, Chile: Centro de Investigación Avanzada en Educación de la Universidad de Chile y el Centro de Estudios de Políticas y Prácticas en Educación de la Pontificia Universidad Católica de Chile, 2012. Disponible en: <http://www.cpeip.cl/usuarios/cpeip/File/ librosestandaresvale/libromediafinal.pdf>. Acceso en: 14 jun. 2016.

Consejo redactores de las Universidades Chilenas. Proyectos de ley MINEDUC. Santiago, Chile: Gobierno de Chile, Ministerio de Educación, 2013. Disponible en: <http:// www.consejoderectores.cl/web/pdf/acta-2013/03/Anexo_47.pdf>. Acceso en: 14 jun. 2016.

Ley General de Educación $N^{\circ} 20.370$ del 17 de agosto de 2009, artículo 46, letra 'g' que establece los criterios de idoneidad del personal docente y no docente en los establecimientos educativos. Santiago, Chile: Biblioteca del Congreso Nacional de Chile, 2009. Disponible en: <http://www.leychile.cl/Navegar/?idNorma=1006043\&idVersion=2009-0912\&idParte>. Acceso en: 14 jun. 2016.

. Marco para la buena enseñanza. Santiago, Chile: Ministerio de Educación, 2008.

NÚNEZZ, I. La identidad de los docentes. Una mirada histórica en Chile. Santiago, Chile: Programa Interdisciplinario de Investigaciones en Educación, 2004.

OSBORNE, D.; GAEBLER, T. Reinventing Government. How the entrepreneurial spirit is transforming the public sector. Nueva York: Plume, 1993.

TENTI, E. Algunas dimensiones de la profesionalización de los docentes: representaciones y temas de la agenda política. R PRELAC, p. 85-103, 2004.

Profesionalización docente: consideraciones sociológicas. En: TENTI, E. (comp.) El oficio de docente: vocación, trabajo y profesión en el siglo XXI. Buenos Aires: Siglo Veintiuno, 2006. p. 119-142.

SISTO, V. Nuevo profesionalismo y profesores: una reflexión a partir del análisis de las actuales políticas de 'profesionalización' para la educación en Chile. Signo y Pensamiento, v. 23, n. 59, p. 178-192, 2011.

THOMPSON, N.; PASCAL, J. Developing critically reflective practice. Reflective Practice, v. 13 n. 2, p. 311-325, 2012.

Recibido en 11 de agosto de 2014.

Aprobado en 23 de mayo de 2016. 